\title{
Effect of applied magnetic field on the rectifying characteristics in self-doped $\mathrm{La}_{0.9} \mathrm{MnO}_{3} / 0.8 \mathrm{wt} \% \mathrm{Nb}-\mathrm{SrTiO}_{3}$ heteroepitaxial junctions
}

\author{
Z. H. Wang, ${ }^{1,2, a)}$ G. L. Yu, ${ }^{1}$ Y. Nie, ${ }^{1}$ Z. F. Wu, ${ }^{1}$ L. Qiu, ${ }^{1}$ Z. Luo, ${ }^{2}$ and J. Gao ${ }^{2}$ \\ ${ }^{1}$ National Laboratory of Solid State Microstructures, Department of Physics, Nanjing University, \\ Nanjiing 210093, People's Republic of China \\ ${ }^{2}$ Department of Physics, The University of Hong Kong, Hong Kong, People's Republic of China
}

(Presented on 9 November 2007; received 28 September 2007; accepted 6 November 2007; published online 4 March 2008)

The epitaxial self-doped $\mathrm{La}_{0.9} \mathrm{MnO}_{3}$ thin film was deposited on a 0.8 wt $\% \mathrm{Nb}$-doped $\mathrm{SrTiO}_{3}$ substrate by pulse laser deposition method to form a bilayer $p-n$ junction with an area of $8 \mathrm{~mm}^{2}$. The isothermal current-voltage loops measured from 10 to $380 \mathrm{~K}$ with an interval of $10 \mathrm{~K}$ in applied magnetic fields up to $7 \mathrm{~T}$ show typical temperature-dependent rectifying characteristic and asymmetrical hysteresis. The effect of magnetic field on the rectifying property is very small for the temperature above $150 \mathrm{~K}$ and a strong asymmetrical effect on the rectifying property below $150 \mathrm{~K}$. In the low temperature region, although the diffusive voltage $V_{d}$ is not affected by the applied magnetic field, the breakdown voltage $V_{b}$ decreases with the increasing of magnetic field. At $10 \mathrm{~K}$, the relative ratio of voltage $\left[V_{b}(0)-V_{b}(7 \mathrm{~T})\right] / V_{b}(0)$ is about $14 \%$, showing a magnetically tunable property. These results reveal the great potential of the manganites in configuring artificial devices. (C) 2008 American Institute of Physics. [DOI: 10.1063/1.2836964]

\section{INTRODUCTION}

The manganite heteroepitaxial junction, showing a good rectifying behavior, was reported by Kawai et al. ${ }^{1,2}$ at first. Due to the high sensitivity of electronic and magnetic properties of manganese to external magnetic field ${ }^{3}$ and electric field, ${ }^{4}$ the variance of resistance and diffusion potential may also make these simple $p$ - $n$ junctions promising for various novel applications. Recently, the effect of magnetic field, ${ }^{5}$ electric field, ${ }^{1}$ and light ${ }^{6}$ on the $p$ - $n$ junctions has been reported. The external field greatly affected the rectifying property and the resistance of the junction, causing an extremely large magnetoresistance. ${ }^{5}$ The magnetically tunable properties of $p-n$ junction is of special interest from the viewpoint of application. In the doped manganites, the carriers dependent on the oxygen content is an open question. Generally, the self-doped $\mathrm{La}_{0.9} \mathrm{MnO}_{3-\delta}$ (LMO) material shows the characteristic of holes. What will be the behavior of the bilayer junction composed of this kind of manganites under external field is an interesting question for us. In this paper, we constructed a bilayer junction using the self-doped LMO film and the 0.8 wt \% Nb-doped $\mathrm{SrTiO}_{3}$ (STON) crystal (LMO/ STON junction), and focused on its behavior under magnetic field. The breakdown voltage $V_{b}$ decreases with the increasing of magnetic field at low temperature region, showing a magnetically tunable property.

\section{SAMPLES AND EXPERIMENTAL}

The epitaxial self-doped $\mathrm{La}_{0.9} \mathrm{MnO}_{3}$ (LMO) thin films $(\sim 100 \mathrm{~nm})$ were deposited on the heating $0.8 \mathrm{wt} \%$

\footnotetext{
${ }^{\text {a) }}$ Author to whom correspondence should be addressed. Also at Department of Physics, Nanjing University, Nanjing 210093, China. Electronic mail: zhwang@nju.edu.cn. Tel.: +8625-8368402. FAX: +8625-83595535.
}

$\mathrm{Nb}-\mathrm{SrTiO}_{3}(\mathrm{STON})$ and $\mathrm{SrTiO}_{3}$ (STO) single crystal substrates $\left(\sim 720^{\circ} \mathrm{C}\right)$ in $1 \mathrm{mbar}$ pure oxygen gas by pulse laser deposition technique and annealed in air at $900{ }^{\circ} \mathrm{C}$ for $1 \mathrm{~h}$. The junction area is about $4 \times 2 \mathrm{~mm}^{2}$. A four-probe method was used to measure the RT curves for LMO/STO sample and the current-voltage $(I-V)$ loops for the manganite heterojunction. For the LMO/STON junction, two evaporated silver electrodes (thickness of $\sim 200 \mathrm{~nm}$ ) are on LMO film and the other two pressed indium electrodes are on STON substrate. In this simple heteroepitaxial junction, the hole conducting LMO is used as the $p$-type region, while the electron conducting STON as $n$-type region. The isothermal $I-V$ loops measured from 10 to $380 \mathrm{~K}$ with an interval of $10 \mathrm{~K}$ in an applied magnetic field up to $7 \mathrm{~T}$ were performed on a Quantum Design physical property measurement system (PPMS9). The measuring current increases from -0.05 to $0.05 \mathrm{~mA}$ then from 0.05 to $-0.05 \mathrm{~mA}$ in a period of $11.2 \mathrm{~s}$ for 256 points. The magnetic field was applied perpendicular to the surface of thin film.

\section{RESULTS AND DISCUSSIONS}

Before we present the behavior of the LMO/STON junction under magnetic field, we display the colossal magnetoresistance (CMR) characteristic of LMO/STO thin film measured at $H=0,0.5,1,3,5$, and $7 \mathrm{~T}$, respectively, as shown in Fig. 1. The temperature-dependent resistivity of the $\mathrm{La}_{0.9} \mathrm{MnO}_{3-\delta}$ thin film under applied magnetic field shows a typical CMR characteristic with metal-insulator transitions at $306.5 \mathrm{~K}$ at $0 \mathrm{~T}$ and $364 \mathrm{~K}$ at $7 \mathrm{~T}$. The temperature of metalinsulator transition increases with the increasing of applied magnetic field. The field dependence of peak temperature $T_{p}^{\mathrm{RT}}$ obtained from RT curves is shown in Fig. 1(b). The field-induced metal-insulator transition temperature increases 


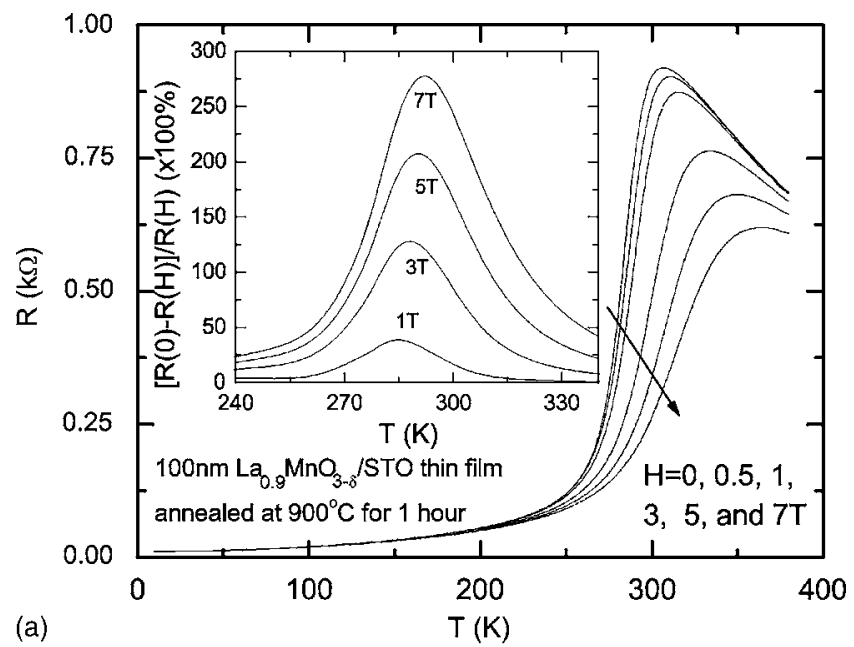

(a)

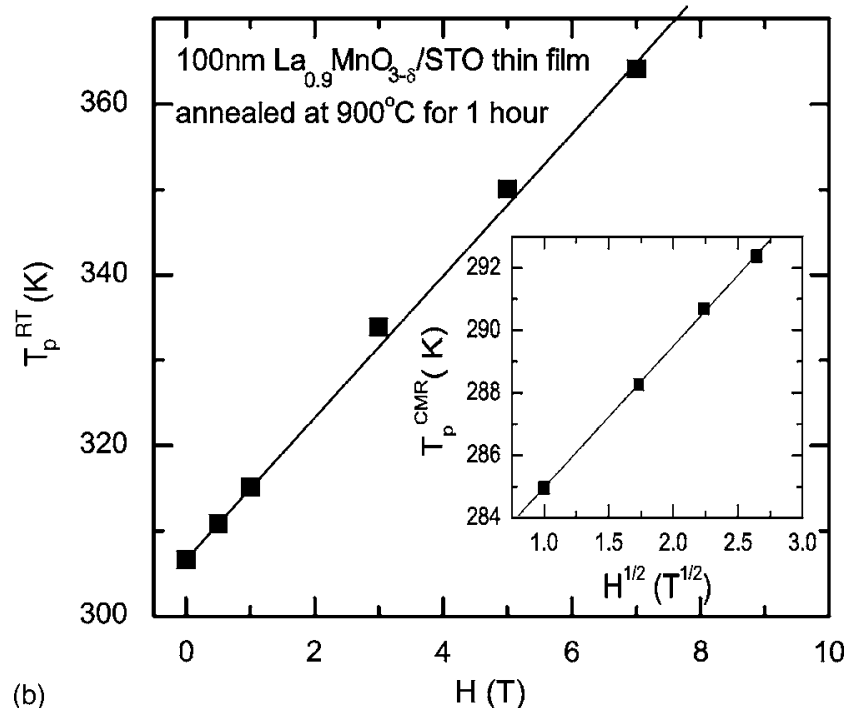

(b)

FIG. 1. Temperature-dependent resistivity of the $\mathrm{La}_{09} \mathrm{MnO}_{3-\delta} / \mathrm{STO}$ film measured at $H=0,0.5,1,3,5$, and $7 \mathrm{~T}$, respectively. They inset displays the magnetoresistance of the film at the several fixed fields. (b) Field dependence of peak temperature $T_{p}{ }^{\mathrm{RT}}$ obtained from RT curves. Inset: field dependence of peak temperature $T_{p}{ }^{\text {CMR }}$ obtained from CMR curves.

linearly with the increasing of applied field. If we defined $\mathrm{MR}=[R(0)-R(H)] / R(H)$, we can calculate the temperaturedependent MR for each field from Fig. 1(a), as shown in the inset of Fig. 1(a). The maximum of MR occurs $289.7 \mathrm{~K}$ at $1 \mathrm{~T}$ and $296 \mathrm{~K}$ at $7 \mathrm{~T}$. The peak temperature $T_{p}{ }^{\mathrm{CMR}}$ obtained from CMR curves increases also with the increasing of applied magnetic field. Our results show that the field dependence of peak temperature $T_{p}^{\mathrm{CMR}}$ follows $T_{p}^{\mathrm{CMR}}(H)$ $=T_{p}^{\mathrm{CMR}}(0)+\alpha H^{1 / 2}$, where $T_{p}^{\mathrm{CMR}}(0)=280.5 \mathrm{~K}$ and $\alpha$ $=4.55 \mathrm{~K} / \mathrm{T}^{1 / 2}$, as shown in the inset of Fig. 1(b). The two peak temperatures show different behaviors.

The isothermal current-voltage loops of the heteroepitaxial LMO/STON junction were measured from 380 to $10 \mathrm{~K}$ with an interval of $10 \mathrm{~K}$ in an applied magnetic field up to $7 \mathrm{~T}$. For clarity, we only show the isothermal $I-V$ loops for $H=0 \mathrm{~T}$ and $7 \mathrm{~T}$, respectively, in Figs. 2(a) and 2(b). From Fig. 2, we can see that all of $I-V$ loops also show a typical rectifying characteristic. As the temperature decreases, the positive part of $I-V$ curve shifts to the right and the negative part to the left. These results indicate that the
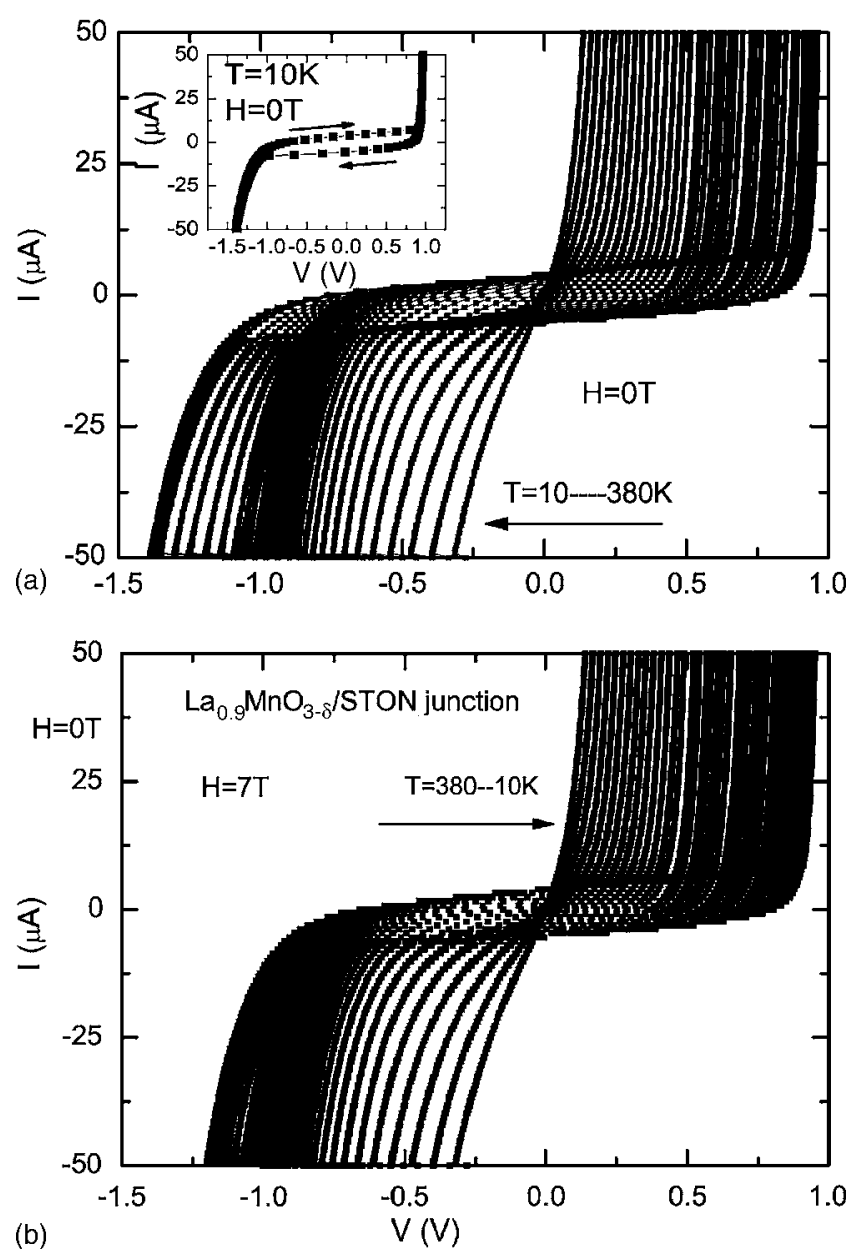

FIG. 2. Isothermal current-voltage loops of the heteroepitaxial LMO/STON junction measured from 380 to $10 \mathrm{~K}$ with an interval of $10 \mathrm{~K}$ in the applied magnetic fields (a) $H=0 \mathrm{~T}$ and (b) $H=7 \mathrm{~T}$, respectively. Inset of Fig. 1(a): isothermal current-voltage loops measured at $10 \mathrm{~K}$ and $0 \mathrm{~T}$.

LMO/STON junction has a $p$ - $n$-like property. Both the diffusive potential $V_{d}$ and the breakdown voltage $V_{b}$ increase with the decreasing of the temperature for the temperature above $50 \mathrm{~K}$. When the temperature is lower than $50 \mathrm{~K}$, the breakdown voltage $V_{b}$ is almost same. In addition, from Fig. 2 , we can see that all of $I-V$ loops do not come from the initial point $(I=0, V=0)$, showing a large asymmetrical hysteretic loop. To see it clearly, we have replotted the $I$ - $V$ curve at $10 \mathrm{~K}$, as shown in the inset of Fig. 1(a). The arrows indicate the increasing and/or decreasing of applied current. The asymmetrically hysteretic $I-V$ loops can be quantitatively simulated by a resistance capacitance shunted junction (RCSJ)-like model $I=I_{0}[\exp (b V / T)-1]+C d V / d t+V / R$. We think that the asymmetrical hysteresis is related with the depletion-layer capacitance of the $p$ - $n$ junction. For a larger area junction, the effect of the junction capacitance on the $I-V$ curve cannot be ignored.

To explore the effect of applied magnetic field on the rectifying behavior, we replot the isothermal current-voltage loops of the heteroepitaxial LMO/STON junction measured at several fixed temperature $T=380$ (magenta lines), 240 (blue lines), 150 (green lines), 100 (red lines) and $10 \mathrm{~K}$ (black lines) for various magnetic fields, respectively, as shown in Fig. 3. From Fig. 3, we can observe that the effect 


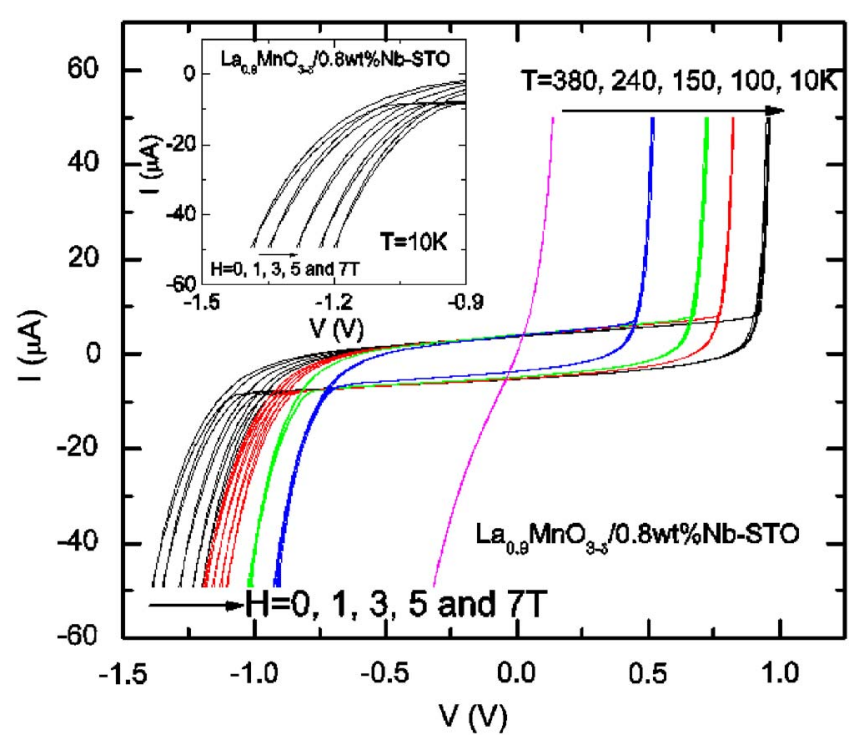

FIG. 3. (Color online) Isothermal current-voltage loops of the heteroepitaxial LMO/STON junction at several fixed temperatures for various applied magnetic fields, respectively. Inset: the negative part of $I-V$ curves measured at $10 \mathrm{~K}$ for $H=0,1,3,5$, and $5 \mathrm{~T}$, respectively.

of magnetic field on the rectifying property is very small for the temperature above $150 \mathrm{~K}$. However, the external field greatly affected the rectifying property below $150 \mathrm{~K}$; meanwhile, the magnetic field has a strong asymmetrical effect on the rectifying property. For the part of positive voltage, the diffusive voltage $V_{d}$ is not affected by the applied magnetic field. However, for the other part, the breakdown voltage $V_{b}$ decreases with the increasing of magnetic field. To see the field effect clearly, we have replotted the negative part of $I-V$ curves measured at $10 \mathrm{~K}$ for various magnetic fields, respectively, as shown in the inset of Fig. 3. Due to the limit of voltage $V=5 \mathrm{~V}$ for $I-V$ measurement in the PPMS, we cannot get the accurate breakdown voltage $V_{b}$. If we defined the voltage at $I=50 \mu \mathrm{A}$ as the breakdown voltage $V_{b}$, we can obtain the field dependence of the breakdown voltage $V_{b}$ at $T=150,100,50$, and $10 \mathrm{~K}$, respectively, as shown in Fig. 4(a). From Fig. 4(a), we can see that the absolute breakdown voltage $V_{b}$ decreases linearly with the applied magnetic field, namely, the breakdown voltage $V_{b}$ is tuned by the applied magnetic field. When the temperature is lower than $50 \mathrm{~K}$, the breakdown voltage $V_{b}$ is independent of the temperature. To show the magnetically tunable properties of the LMO/STON $(p-n)$ junction clearly, we calculate the relative ratio of breakdown voltage $\left[V_{b}(0)-V_{b}(7 \mathrm{~T})\right] / V_{b}(0)$ at fixed temperature for various magnetic fields from the data of Fig. 4(a). The field dependence of the relative ratio of breakdown voltage is shown in Fig. 4(b). At $10 \mathrm{~K}$, the relative ratio of voltage $\left[V_{b}(0)-V_{b}(7 \mathrm{~T})\right] / V_{b}(0)$ is about $14 \%$. These results reveal the great potential of the manganites in configuring artificial devices.

\section{CONCLUSIONS}

The epitaxial self-doped $\mathrm{La}_{0.9} \mathrm{MnO}_{3} / 0.8 \mathrm{wt} \% \mathrm{Nb}-$ $\mathrm{SrTiO}_{3}$ junction shows a typical temperature-dependent rec-
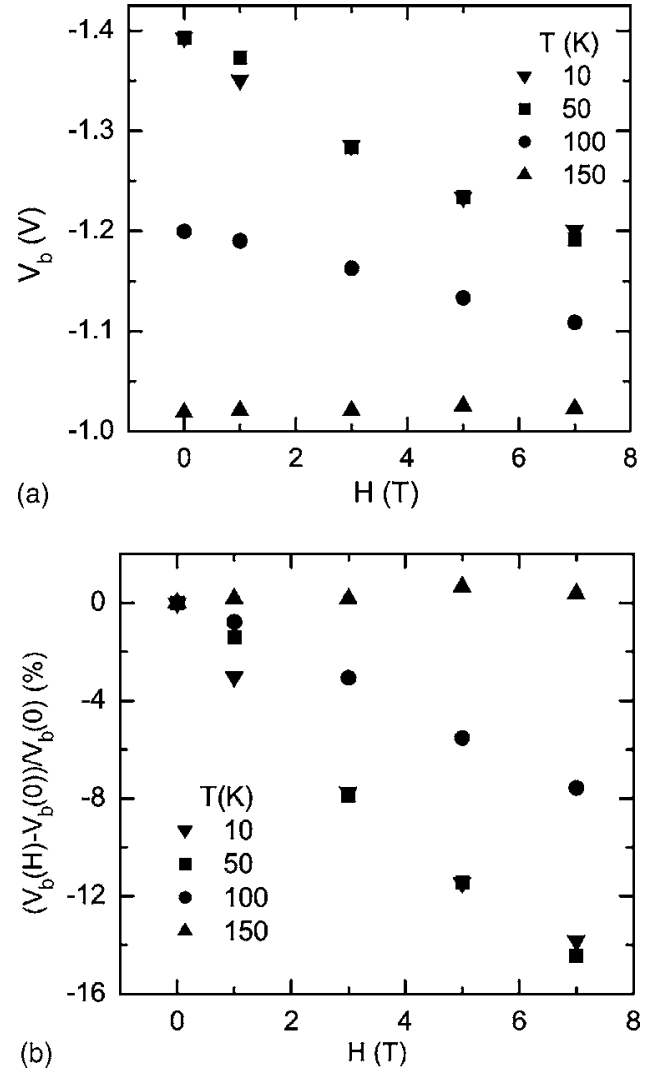

FIG. 4. (a) Field dependence of the breakdown voltage $V_{b}$ at $T=150,100$, 50 , and $10 \mathrm{~K}$, respectively. (b) Field dependence of the breakdown voltage $V_{b}$ ratio $\left[V_{b}(H)-V_{b}(0)\right] / V_{b}(0) * 100 \%$ at $T=150,100,50$, and $10 \mathrm{~K}$, respectively.

tifying characteristics and asymmetrical hysteresis. Above $150 \mathrm{~K}$, the effect of magnetic field on the rectifying property is very small. However, the magnetic field has a strong asymmetrical effect on the rectifying property below $150 \mathrm{~K}$. The breakdown voltage $V_{b}$ decreases with the increasing of magnetic field, showing a magnetically tunable. At $10 \mathrm{~K}$, the relative ratio of voltage $\left[V_{b}(0)-V_{b}(7 \mathrm{~T})\right] / V_{b}(0)$ is about $14 \%$ at $7 \mathrm{~T}$. The magnetic field effect maybe related with the spin glass state. These results reveal the great potential of the manganites in configuring artificial devices.

\section{ACKNOWLEDGMENTS}

The authors acknowledge partial support from the Research Grant Council of Hong Kong (Project No. HKU 7024/05P).

${ }^{1}$ H. Tanaka, J. Zhang, and T. Kawai, Phys. Rev. Lett. 88, 027204 (2002).

${ }^{2}$ J. Zhang, H. Tanaka, and T. Kawai, Appl. Phys. Lett. 80, 4378 (2002).

${ }^{3}$ S. Jin, T. H. Tiefel, M. McCormack, R. A. Fastnacht, R. Ramesh, and L.

H. Chen, Science 264, 413 (1994).

${ }^{4}$ A. Asamitsu, Y. Tomioka, H. Kuwahara, and Y. Tokura, Nature (London) 388, 50 (1997).

${ }^{5}$ J. R. Sun, C. M. Xiong, T. Y. Zhao, S. Y. Zhang, Y. F. Chen, and B. G. Shen, Appl. Phys. Lett. 84, 1528 (2004).

${ }^{6}$ Z. Luo and J. Gao, J. Appl. Phys. 100, 056104(R) (2006). 